Proc. 13th Econophysics Colloquium (EC) and 9th Symposium of Physics in Economy and Social Sciences (FENS), 2017

\title{
Tight-Binding Approach: A Quantum Mechanical Tool to Study Economic Time Series
}

\author{
H. CRUZ* \\ Departamento de Fisica, Universidad de La Laguna, Avda. Astrofisico Francisco Sanchez, s/n, \\ 38204 La Laguna, Tenerife, Spain
}

Presented also at 13th Econophysics Colloquium 2017 (EC 2017)

\begin{abstract}
We study the localization properties of the 1D tight-binding equation, where the on-site potential is aperiodic or pseudorandom. The on-site potential values are derived from economic time series databases. We carry out numerical work involving direct diagonalization to study localization properties of the system. In our model, eigenstates at the band center are all extended whereas the band-edge states are all localized. This diagonalization scheme is applied to different segments of the time series. The Lyapunov exponent behaves at $E=0$ as $\gamma(E) \sim|E|^{\beta}$. The results lead us to conclude that this mathematical tool could be used as a moving indicator to study economic charts.
\end{abstract}

DOI: $10.12693 /$ APhysPolA.133.1347

PACS/topics: 72.15.Lh, 72.15.Rn, 89.65.Gh

\section{Introduction}

Since long time ago, scientists have tried to infer future states of dynamical systems. The prediction of such systems is of great importance in all scientific areas. These studies include topics as diverse as weather forecasting, the estimation of quantum evolution, statistics, econophysics [1] or the prediction of social behavior.

Quantum mechanics can provide a theoretical frame to model socioeconomic systems. In the last years, the Hamiltonian formulation of quantum finance (QF) and the random matrix theory (RMT) has been applied to finance [2]. Quantum finance refers to the application of the mathematical formalism of quantum mechanics to problems arising in finance. The Hamiltonian formulation of quantum finance is an area of active research. Using quantum finance, the theory of coupon bond and interest rate options have been studied extensively [3].

In the decade of fifties, quantum random matrix theory (QRMT) was developed to provide a framework for the study of quantum many-body systems. Since being reported [4], RMT has been the object of much attention due to its possible application to finance time series [5].

Using this bridge, many techniques developed along the years can be transferred to study economic time series. Utilizing this approach, we analyze in detail a tightbinding model in which the on-site potential values are derived from finance data.

\section{Model}

In condensed matter physics, the approach that starts out from the wave functions of the free atoms is known as

\footnotetext{
* corresponding author
}

linear combination of atomic orbitals or tight-binding approximation. Tight-binding method is applied to a wide variety of solids [6]. Electrons in this model are tightly bound to the atom to which they belong (Fig. 1). Due to the interaction with potentials and states on neighboring atoms is limited, the wave function of the electron will be rather similar to the atomic orbital of the free atom to which it belongs [7].

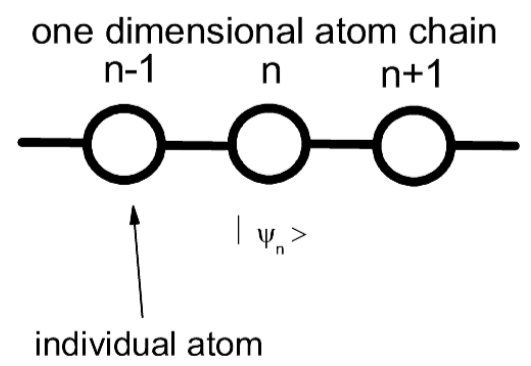

Fig. 1. Schematic drawing of a 1D atom chain. Charge distributions of adjacent atoms overlap when the atoms are brought together to form a one-dimensional crystal.

The one-dimensional nearest-neighbor tight-binding model is described by the equation

$$
u_{n+1}+u_{n-1}+V_{n} u_{n}=E u_{n},
$$

where the strength of the nearest-neighbor hopping has been set equal to unity. In Eq. (2.1), $u_{n}$ is the amplitude of the electron wave function at the $n$ lattice site with $V_{n}$ as the on-site potential. $E$ is the electron eigenvalue. A $V_{n}$ periodic in $n$ has all its states extended (the Bloch states).

Before developing the model, we introduce two related quantities: the density of states $D(E)$ and the Lyapunov exponent $\gamma(E)$. The density of states is 


$$
D(E)=\sum_{j} \delta\left(E-E_{j}\right),
$$

where $E_{j}$ are the eigenenergies. In addition, the localization length of wave functions follows the equation:

$$
\left|\psi_{j}(x)\right| \sim \mathrm{e}^{-x / \xi_{j}},
$$

where $\xi_{j}$ is the localization length. The Lyapunov exponent is seen to be the inverse localization length,

$$
\gamma_{j}=\frac{1}{\xi_{j}} \text {. }
$$

There are two equations of particular interest in the localization problems

$$
\gamma_{j}\left(E_{j}\right)=\frac{1}{N-1} \sum_{j \neq l} \ln \left|E_{j}-E_{l}\right|
$$

and

$$
\gamma_{j}\left(E_{j}\right)=\frac{1}{N-1} \sum_{j \neq l} \ln \left|\frac{u_{n+1}}{u_{n}}\right|,
$$

where $N$ is the number of atomic sites. Equations (2.5) and (2.6) are useful in obtaining the Lyapunov exponent.

Then, the behavior of the Lyapunov exponent at the mobility edge can be written as

$$
\gamma(E) \sim\left|E-E_{c}\right|^{\beta}
$$

around $E>E_{c}$

This model has been developed to study a different class of one-dimensional potentials which are neither periodic nor random. These potentials are deterministic and aperiodic, and are described by the equation

$$
V_{n}=\lambda \cos \left(\pi \alpha n^{\nu}\right),
$$

where $\alpha$ is a real number and $\lambda$ is the strength of the potential having a value between 0 and $2 . \alpha$ is a real number and $\nu$ is taken to lie between 0 and 1 . Equation (2.8) describes a very slowly varying potential. The slow spatial variation of $V_{n}$ is crucial in producing the localization properties of the model. For $0<\nu<1$ and $0<|\lambda|<2$, Das Sarma et al. found the existence of a mobility edge in this one-dimensional model [8].

In general, one-dimensional models do not allow for the existence of mobility edges. The random Anderson model produces only localized electron states in one dimension for all energies $[9,10]$. The incommensurate Harper's equation has not mobility edges either, with all states localized or extended. The existence of mobility edges in Eq. (2.8) is unique in the sense that it is the only known gapless potential allowing for the existence of a metal-insulator transition.

The potential defined by Eq. (2.8) has extended states at the band center $\left(-E_{c}<E<E_{c}\right)$ and localized states at the band edges $\left(|E|>E_{c}\right)$. The mobility edges are at $\pm E_{c}= \pm(2-|\lambda|)$. The unperturbed tightbinding model $(\lambda=0)$ has absolute band edges at \pm 2 , whereas the perturbed model has absolute band edges at $\pm E_{c}= \pm(2-|\lambda|)$.

The potential defined by Eq. (2.8) has long-range coherence. The nature of metal-insulator transition in this potential is substantially different from the usual Anderson-localization problem. We can notice that a
$V_{n}$ random potential produces all localized states in onedimension (the Anderson model). As $n$ increases, this potential becomes more and more slowly varying. For $\nu \geq 2, V_{n}$ is pseudorandom and Eq. (2.8) becomes equivalent to the random Anderson model producing only localized states in 1D. Thouless [11] showed that all states (except exactly at $E_{c}=0$ ) are localized at $1<\nu<2$. The Lyapunov exponent vanishes very slowly as one approaches the band center.

The Lyapunov of the class of one-dimensional potentials being studied in this paper is allowed to have mobility edges at $E_{c}=0(\lambda=2)$. It is found that the exact nature of the divergence depends on the particular form of the on-site potential $V_{n}$. In this work, we shall use this property to try to forecast finance time series.

One can use the successive differences of the natural logarithm of price $P(n)$. Then, the $V_{n}$ potential is modified as follows:

$$
V_{n}^{\prime}=\lambda \cos \left(\pi \alpha n^{\nu}\right)+\vartheta \ln \frac{P(n+1)}{P(n)},
$$

where $\vartheta$ is an adimensional coefficient taken to be $\vartheta=50$. The price correction to the potential is about $10 \%$. In our case, the economic time series database is the EURUSD daily price from March 2011 to October 2017 (Fig. 2).

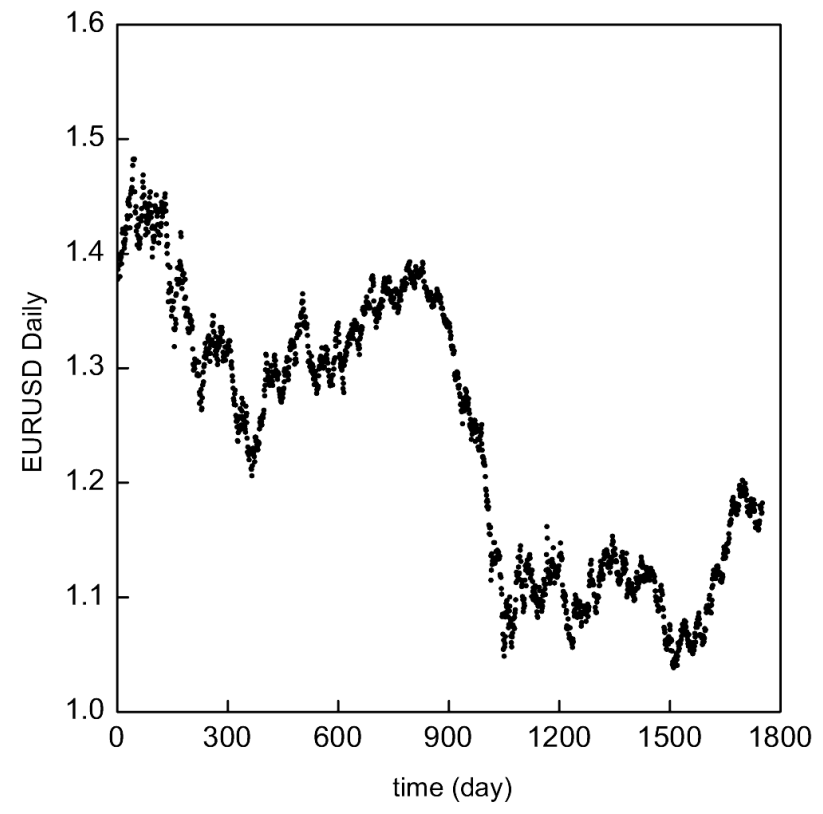

Fig. 2. EURUSD Daily versus time.

We shall try to explain the role of the second term of Eq. (2.9), the $\vartheta$ term (the market term). The market term acts as a disorder term, i.e., the typical random potential present in localization problems. The tightbinding approach has been widely used to study localization problems considering random and incommensurate potentials [8]. We can also notice that periodic $V_{n}$ is the usual Bloch case whereas Eq. (2.1) with a random potential is the Anderson model. A random $V_{n}$ can be used with no loss of generality. 
The first term on the right-hand side of Eq. (2.9) is the only known gapless potential allowing for the existence of a metal insulator transition in one dimension. It is an incommensurate potential [8]. The market term $(\vartheta$ term) acts as disorder term in our model. In this way, the market behavior will be reflected in the localization properties of the total potential due to the specific properties of the incommensurate potential, first term on the right-hand side of Eq. (2.9). The $\vartheta$ coefficient value has been taken as $\vartheta=50$ to have visible results.

\section{Results}

We have studied the localization properties of a class of $1 \mathrm{D}$ tight-bindings models where the on-site diagonal potential is $V_{n}^{\prime}$ :

$$
u_{n+1}+u_{n-1}+V_{n}^{\prime} u_{n}=E u_{n} .
$$

Our calculation involves direct diagonalization of the Hamiltonian to directly calculate the eigenenergies. This diagonalization scheme are applied to different segments of the time series. In our case, the segment size $N_{s}$ is 400 points.

The Lyapunov exponent $\gamma(E)$ for our model as well as the critical exponent $\beta$ defined by the relations $\gamma(E) \sim$ $\left|E-E_{c}\right|^{\beta}$ can be easily obtained. In Fig. 3 we show our numerical results for $\gamma(E)$ which clearly shows the existence of mobility edges (and a metal-insulator transition) at $E= \pm 1.6$.

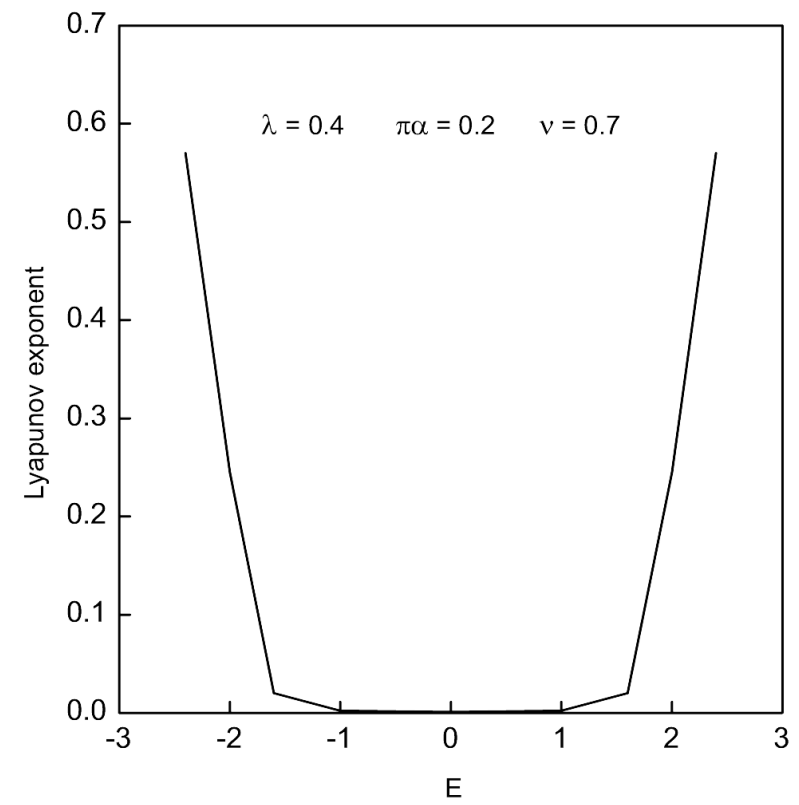

Fig. 3. The Lyapunov exponent versus energy for the potential defined in Eq. (2.8). We can see that the mobility edges are at $E_{c}=-1.6$ and $E_{c}=1.6$.

In Fig. 4 we plot our calculated Lyapunov exponent for the $V_{n}$ and $V_{n}^{\prime}$ potentials by changing $\lambda$ to $\lambda=2.0$, keeping the other parameters the same $(\pi \alpha=0.2$ and $\nu=0.7)$. The two mobility edges at \pm 1.6 now merge and shift to the center of the band at $E_{c}=0$. The Lyapunov exponent vanishes very slowly at the band center. The segment size is $N_{s}=400$.

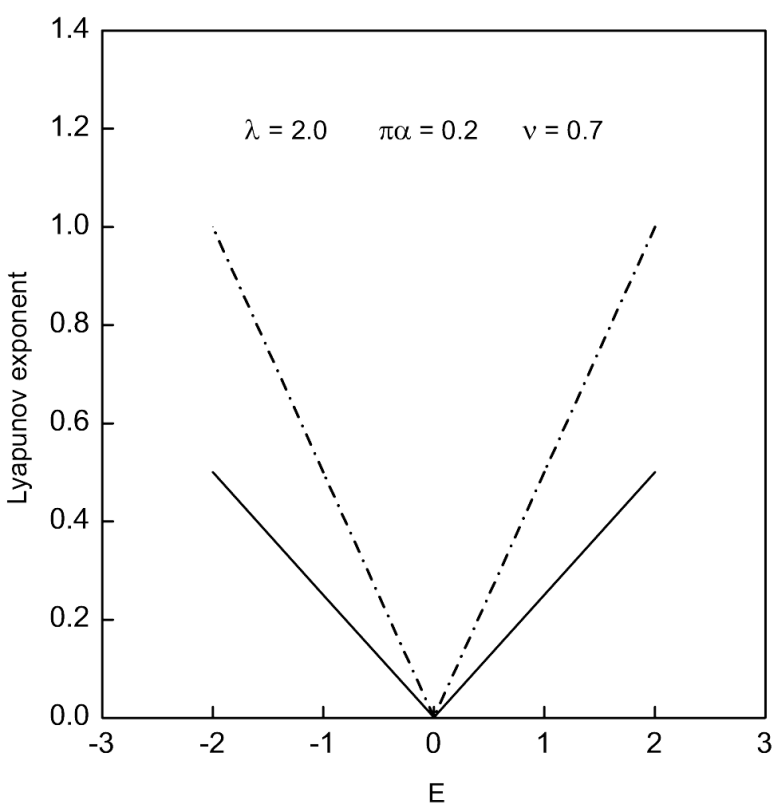

Fig. 4. The Lyapunov exponent versus energy for the potential defined in Eq. (2.8). We can see that the mobility edges are at $E_{c}=0$. Solid line: $V_{n}$ potential. Dashed line: $V_{n}^{\prime}$.

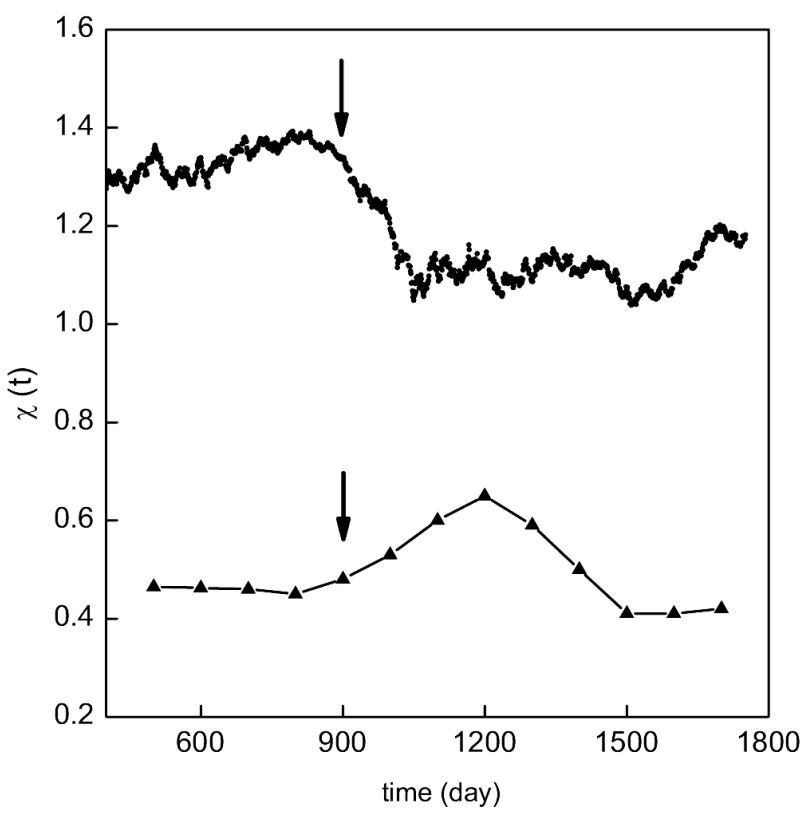

Fig. 5. $\chi$ versus time (day). We have also plotted EURUSD daily price. 
Examining Fig. 4, we discover that we can easily obtain the slope of $\gamma(E)$,

$$
\chi(E)=\frac{\mathrm{d} \gamma(E)}{\mathrm{d} E} .
$$

The time series is divided into $N / N_{s}$ smaller segments being $N$ the time series size. Then, we calculate $\chi(t)$ for each segment. If we obtain the $\chi(t)$ value every $N_{s}$ points, we have a moving indicator.

Figure 5 shows $\chi(t)$ versus time (day). We have also plotted EURUSD daily price. If the price is decreased, the $\chi$ value will be increased (see arrows in Fig. 5) due to the $V_{n}^{\prime}$ potential contribution, Eq. (2.9). Then, the $\chi$ value decreases again as time progresses. As a result, this mathematical tool could be used as a moving indicator to study economic charts.

In summary, we analyze the localization properties of the one-dimensional tight-binding equation, where the on-site potential is derived from finance data. A diagonalization scheme is applied to different segments of the time series. We have shown the possibility of having a new moving indicator based on a quantum mechanical tool. The specific properties of the right-hand side of Eq. (2.9), i.e. the incommensurate potential, can reflect market changes.

\section{References}

[1] R.N. Mantegna, H.E. Stanley, An Introduction to Econophysics: Correlations and Complexity in Finance, Cambridge University Press, 2000.

[2] B.E. Baaquie, Phys. Rev. E 77, 036106 (2008).

[3] B.E. Baaquie, Quantum Finance, Cambridge University Press, 2004.

[4] V. Pleron, P. Gopikrishnan, B. Rosenow, L.A. Nunes Amaral, H.E. Stanley, Phys. Rev. Lett. 83, 1471 (1999).

[5] F.G. Pedro, A. Westphal, Phys. Rev. E 95, 032144 (2017).

[6] P.W. Anderson, Phys. Rev. 109, 1492 (1958).

[7] D.J. Thouless, Phys. Rep. 13, 95 (1974).

[8] S. Das Sarma, S. He, X.C. Xie, Phys. Rev. B 41, 5544 (1990).

[9] H. Cruz, S. Das Sarma, J. Phys. I (France) 3, 1515 (1993).

[10] H. Cruz, J. Appl. Phys. 113, 153706 (2013).

[11] D.J. Thouless, Phys. Rev. Lett. 61, 2141 (1988). 